ALÉTHEIA

Enero - Diciembre. 2019, Vol. N7, pp. 53-61

DOI: 10.33539/aletheia.2019.n7.2153

\section{COMPETENCIAS DIGITALES EN ESTUDIANTES DEL SÉPTIMO CICLO DE EDUCACIÓN SECUNDARIA A TRAVÉS DE LA RED SOCIAL EDUCATIVA EDMODO}

\author{
DIGITAL LITERACY IN STUDENTS FROM \\ THE SEVENTH CYCLE OF HIGH SCHOOL \\ EDUCATION THROUGH EDUCATIONAL \\ SOCIAL NETWORK EDMODO
}

\section{RESUMEN}

El propósito de la investigación fue conocer el nivel de desarrollo de las competencias digitales alcanzado por los estudiantes de $\mathrm{III}^{\circ}$, $\mathrm{IV}^{\circ}$ y $\mathrm{V}^{\circ}$ de secundaria de una institución educativa pública de Lima Metropolitana mediante actividades propuestas en la red social educativa Edmodo. Para ello, se elaboró un cuestionario sobre la base del Inventario de Competencias TIC con la finalidad de recoger información acerca de las competencias digitales de los estudiantes antes y después de la intervención. El instrumento se aplicó a una muestra de 162 estudiantes divididos en dos grupos: uno de control (80) y uno experimental (82). Los resultados evidencian que el segundo grupo alcanzó un nivel medio en la variable "competencias digitales", cuya subvariable "conocimiento de herramientas digitales" obtuvo mejores resultados.

Palabras clave: Competencias digitales, red social educativa Edmodo, conectivismo.

\section{ABSTRACT}

The purpose of this study was to establish the level of digital literacy in students from 3rd, 4th and 5th grade of a public high school in Lima, by a group of activities in the educational social network Edmodo. In order to collect information about the digital literacy of students, before and after the intervention, it has developed a questionnaire based on the ICT Competency Inventory. The instrument was applied to a sample of 162 students divided into two groups: one control group (80) and one experimental group (82). The results showed that the second group achieved a medium level in the variable "digital literacy", in which the "knowledge of digital tools" outperformed.

Keywords: Digital literacy, educational social network Edmodo, connectivism
Mg. Mónica Gladys Chapilliquén Rodríguez mchapilliquenr@pucp.pe

Pontificia universidad Católica del Perú

\section{INTRODUCCIÓN}

En la actualidad, es usual observar a los adolescentes interactuar con naturalidad a través de las redes sociales. Al igual que los miembros de la actual "sociedad red", denominada así por Castells (2006), en la que todos estamos inmersos, ellos se encuentran constantemente conectados a través de diferentes medios tecnológicos. Esto ha generado nuevas formas de comunicación sustentadas por la teoría del conectivismo (Siemens, 2004) y nuevas estrategias de aprendizaje basadas en entornos virtuales sustentadas por el socioconstructivismo (Jonnaert, 2001). En ese sentido, el uso pedagógico de las herramientas tecnológicas online y, en particular de la red social educativa Edmodo, se convierten en recursos promisorios para el desarrollo de las competencias digitales en los estudiantes, tal como se demuestra en la presente investigación. Por esta razón, el propósito del presente artículo es presentar el nivel de desarrollo de las competencias digitales alcanzado por los estudiantes del séptimo ciclo del nivel secundaria $\left(\mathrm{III}^{\circ}, \mathrm{IV}^{\circ} \mathrm{y} \mathrm{V}^{\circ}\right)$, de una institución educativa pública de Lima Metropolitana, mediante el uso de la red social educativa Edmodo. 


\section{Fundamentación}

\subsection{Razones para integrar las redes sociales en el aula}

El uso de las redes sociales educativas responde al paradigma tecnológico asumido por la sociedad de la información y la comunicación. En ella, la cultura de la virtualidad se impone y se caracteriza por el uso de la información en la generación del conocimiento, además, por la morfología de la red que responde a la lógica de interconexión. Así, la participación en una red social se presenta como una nueva forma de comunicación y de construcción del conocimiento escolar (Aguaded y Cabero, 2002), ya que responde a la tecnología que caracteriza a la revolución tecnológica (Suárez, 2008). Por esta razón, una educación en red, basada en el uso de redes sociales, constituye una innovación pedagógica ya que se utilizan recursos acordes con la realidad del estudiante.

La teoría del conectivismo explica el proceso de aprendizaje con mediación tecnológica, a diferencia de las teorías conductista, cognitivista y constructivista, que se mantienen al margen. Los principios del conectivismo propuestos por Siemens (2004) enfatizan que el aprendizaje se produce a través de redes de conexión; siendo la persona o institución el punto de partida o nodo de interacción en la red. Estas conexiones son una oportunidad educativa, porque generan nuevos espacios de aprendizaje a los cuales los adolescentes acceden para interactuar, procesar y compartir información. En ese sentido, el socioconstructivismo emerge como una teoría dentro del enfoque constructivista, en el que la interacción social adquiere mayor importancia, debido a que los vínculos afectivos, sociales y contextuales contribuyen a la construcción personal del conocimiento (Jonaert, 2001). Por esta razón, los docentes deben reconocer que las redes sociales son espacios favorables para el desarrollo de competencias digitales.

\subsection{Las competencias digitales}

La competencia digital es al mismo tiempo una necesidad y un derecho de todos los ciudadanos (Ferrari, 2012). En ese sentido, los docentes y los estudiantes necesitan desarrollarlas para adaptarse a los entornos influenciados por tecnología. Una persona es digitalmente competente cuando comprende el funciona- miento de los recursos multimedia, sabe buscar información, es crítica frente a qué elegir o no de internet, y se comunica por medio de diversas herramientas y aplicaciones (Ferrari, 2012). En la misma línea, Vivancos (2008) presenta los siguientes tres aspectos fundamentales en la competencia digital: el informacional, el audiovisual y el informático, así como sus seis dimensiones clave: la cognitiva, la colaborativa, la comunicacional, la creativa, la ética y la instrumental. Por otro lado, el desarrollo de competencias digitales responde a la visión de que la escuela debe formar para aprender a lo largo de toda la vida y para participar en la llamada sociedad de la información (Adell, 2011; Calvani, Fini y Ranieri, 2010). Luego, Adell (2011) propone los siguientes cinco componentes de la competencia digital: la competencia informacional, la competencia tecnológica, la alfabetización múltiple, la competencia cognitiva genérica y la ciudadanía digital.

Para el presente estudio, la competencia digital es el conjunto de conocimientos, habilidades y actitudes para buscar, obtener, procesar y comunicar información en diferentes formatos digitales a través de las TIC. Esto implica un cambio en la concepción del proceso de enseñanza-aprendizaje por parte del docente, concepción reflejada en la opción por una metodología de trabajo colaborativo y en red. De la misma manera, implica la adaptación de los estudiantes al uso de herramientas tecnológicas, la capacidad para trabajar colaborativamente, la habilidad para recrear información y difundirla a través de la red, la capacidad para analizar y sintetizar grandes flujos de información simultánea y la perseverancia en un comportamiento ético al trabajar en entornos virtuales. Morduchowicz (2012) afirma que estas habilidades los convierten progresivamente en seres productores de conocimiento.

En ese sentido, se formularon cinco subvariables de la competencia digital. El conocimiento de las herramientas digitales está referido a la capacidad para informarse acerca del funcionamiento de Mindomo, Animoto, Voki, Easel.ly, Pixton y Edmodo. El uso de las herramientas digitales está considerado como la aplicación de estas en actividades con o sin propósitos educativos. El acceso y procesamiento de la información digital están referidos a la capacidad para obtener información de manera eficiente, en breve tiempo y 
de fuentes confiables; y de analizar textos, imágenes, audios, títulos, sub títulos y esquemas gráficos. En la ética en el uso de la información digital están consideradas las actitudes de respeto y uso responsable durante el trabajo colaborativo en los entornos virtuales; además, del respeto a la propiedad intelectual. Finalmente, la comunicación de la información en diferentes formatos digitales implica la capacidad de expresarse a través de un video (Animoto), una infografía (Easel.ly), una historieta (Pixton), un audio (Voki) o un organizador visual (Mindomo).

\subsection{Edmodo, la red social educativa para el desa- rrollo de competencias digitales}

Las redes sociales gozan de una excepcional capacidad de contactar a las personas (De Haro, 2011). Esto hace posible que los usuarios, quienes comparten perfiles personales o profesionales, se comuniquen, compartan información y consoliden las comunidades establecidas (Avalos, 2013; Castañeda, 2010). Debido a su potencial comunicativo las redes sociales pueden ser aprovechadas como recursos educativos alternativos, ya que incrementan el nivel de conectividad de los participantes y promueven la formación de comunidades de aprendizaje (Vásquez-Martínez y Cabero-Almenara, 2015).

Edmodo, creada en el año 2008 por Jeff O'Hara y Nick Borg, "es una plataforma educativa que brinda un entorno amigable y sencillo, que se puede utilizar para generar un aula virtual, en la que los profesores y los alumnos pueden compartir contenidos, actividades, generar debates" (Avalos, 2013, p. 77). Los docentes pueden crear grupos, subgrupos, administrar calificaciones, compartir recursos multimedia, formular encuestas, asignar tareas y organizar un calendario. Los estudiantes pueden comentar los trabajos de sus pares, subir y descargar archivos, responder encuestas, y publicar sus producciones individuales o colectivas. Finalmente, los padres de familia pueden acceder al grupo al que pertenecen sus hijos y verificar sus avances y logros durante el proceso de aprendizaje. Todos pueden acceder a esta red social desde una computadora, un smartphone o un iPhone a través de la URL $<$ www.edmodo.com $>$.

Respecto al uso de Edmodo, se han producido distintas experiencias educativas. Por ejemplo, Vásquez (2013) la usa para evidenciar el desarrollo de competencias básicas en estudiantes de secundaria; Sáez, Lorraine y Miyata (2013) la utilizan para el desarrollo de proyectos colaborativos en primaria; y Sáez, Fernández y García (2012) la utilizan en la educación de adultos. En todos los casos, los resultados son óptimos e invitan a ampliar el campo de aplicación de este entorno virtual.

La presente investigación tuvo los siguientes objetivos: (1) reconocer el nivel de desarrollo de las competencias digitales que presentan los estudiantes del séptimo ciclo de secundaria, antes de utilizar la red social educativa Edmodo; y (2) determinar el nivel de desarrollo de las competencias digitales alcanzado por los estudiantes del séptimo ciclo de secundaria luego de utilizar la red social educativa Edmodo. De la misma manera, se pretendió demostrar la hipótesis general de si el nivel de desarrollo de las competencias digitales que alcanzan los estudiantes del grupo experimental en el postest difiere significativamente del nivel presentado por el mismo grupo en el pretest. Además, se pretendió comprobar las siguientes subhipótesis: (1) si el nivel de desarrollo de las competencias digitales que presentan los estudiantes del grupo de control difiere significativamente del nivel presentado por el grupo experimental antes; y (2) después del uso de la red social educativa Edmodo.

\section{Metodología, enfoque, nivel y diseño de la in- vestigación}

La investigación responde al enfoque cuantitativo, porque se explicó un fenómeno educativo según los pasos del método científico y se trabajó con una muestra y datos cuantitativos (Hernández, Fernández y Baptista, 2010; Bisquerra, 1989). La muestra fue seleccionada de forma no probabilística e intencional, debido a que los grupos de control y experimental eran intactos y se habían formado antes de la investigación (Sánchez y Reyes, 2009; Hernández et al., 2010; Creswell, 2014). El nivel de investigación fue explicativo o de comprobación de hipótesis causales (Sánchez y Reyes, 2009), debido a que se formularon hipótesis que fueron sometidas a comprobación mediante métodos estadísticos. El diseño de la investigación fue cuasiexperimental, con pretest y postest y con grupo de control y grupo experimental (Bisquerra, 1989; Hernández, et al., 2010; Vogt, 2007). 
La población estuvo conformada por 295 estudiantes de las secciones "A", "B", "C", y "D" del III', $\mathrm{IV}^{\mathrm{o}}$ y $\mathrm{V}^{\mathrm{o}}$ de secundaria de ambos turnos. Esta se dividía en 3 secciones "A" del $\mathrm{III}^{\mathrm{o}}, \mathrm{IV}^{\mathrm{o}}$ y $\mathrm{V}^{\mathrm{o}}$ y 3 secciones "B" de los mismos grados en el turno mañana (162 estudiantes). De la misma forma se distribuyeron las secciones " $C$ " y " $D$ " de los mismos grados en el turno tarde (133 estudiantes). La muestra estuvo conformada 162 estudiantes: 82 de las secciones " $\mathrm{A}$ " del III', IV y V (grupo experi- mental) y 80 de las secciones "B" del III', $\mathrm{IV}^{\mathrm{o}}$ y V (grupo de control).

\subsection{Recopilación de datos}

Para alcanzar los objetivos propuestos se aplicó un cuestionario de competencias digitales adaptado del Inventario de Competencias TIC (Incotic) en su versión Incotic - ESO (2012). Los ítems elaborados se encuentran agrupados de la siguiente manera:

Tabla 1. Distribución de los ítems del cuestionario de competencias digitales

\begin{tabular}{clr}
\hline Cuestionario de Competencias Digitales (campos temáticos) & Número de \\
\hline 1. Conocimiento de las herramientas digitales & 18 \\
2. & Uso de las herramientas digitales & 14 \\
3. Acceso y procesamiento de la información digital & 19 \\
4. Ética en el uso de la información digital & 05 \\
5. Comunicación de la información en diferentes formatos & 08
\end{tabular}

TOTAL 64

Fuente: elaboración propia La escala de medida de tipo ordinal se distribuye en tres rangos: bajo, medio y alto (Vogt, 2007), los que se muestran en la siguiente tabla:

Tabla 2. Escala de medida del nivel de competencia digital

\begin{tabular}{|c|c|c|c|c|c|}
\hline \multicolumn{5}{|c|}{ Subvariables } & \multirow{2}{*}{$\begin{array}{c}\text { Variable } \\
\text { Competencia } \\
\text { digital }\end{array}$} \\
\hline $\begin{array}{l}\text { Conocimiento } \\
\text { herramientas }\end{array}$ & $\begin{array}{c}\text { Uso de } \\
\text { herramientas }\end{array}$ & $\begin{array}{c}\text { Acceso y } \\
\text { procesamiento }\end{array}$ & $\begin{array}{l}\text { Ética en el } \\
\text { uso inform. }\end{array}$ & $\begin{array}{c}\text { Com. En } \\
\text { formatos dig. }\end{array}$ & \\
\hline $\begin{array}{c}\text { Nivel bajo: } \\
0-5\end{array}$ & $\begin{array}{c}\text { Nivel bajo: } \\
0-18\end{array}$ & $\begin{array}{c}\text { Nivel bajo: } \\
0-5\end{array}$ & $\begin{array}{l}\text { Nivel bajo: } \\
0-6\end{array}$ & $\begin{array}{c}\text { Nivel bajo: } \\
0-10\end{array}$ & $\begin{array}{c}\text { Nivel bajo: } \\
0-64\end{array}$ \\
\hline $\begin{array}{c}\text { Nivel medio: } \\
6-11\end{array}$ & $\begin{array}{c}\text { Nivel medio: } \\
19-37\end{array}$ & $\begin{array}{c}\text { Nivel medio: } \\
26-51\end{array}$ & $\begin{array}{c}\text { Nivel medio: } \\
7-13\end{array}$ & $\begin{array}{c}\text { Nivel medio: } \\
11-21\end{array}$ & $\begin{array}{c}\text { Nivel medio: } \\
65-113\end{array}$ \\
\hline $\begin{array}{c}\text { Nivel alto: } \\
12-18\end{array}$ & $\begin{array}{c}\text { Nivel alto: } \\
38-56\end{array}$ & $\begin{array}{l}\text { Nivel alto: } \\
52-77\end{array}$ & $\begin{array}{l}\text { Nivel alto: } \\
14-20\end{array}$ & $\begin{array}{l}\text { Nivel alto: } \\
22-32\end{array}$ & $\begin{array}{l}\text { Nivel alto: } \\
134-203\end{array}$ \\
\hline
\end{tabular}

Fuente: elaboración propia

En consecuencia, según el puntaje obtenido, los estudiantes se ubicaron en uno de los siguientes niveles: Nivel bajo $=1$ Nivel medio $=2$ Nivel Alto $=3$

\subsection{Validación de instrumentos}

El cuestionario de competencias digitales fue validado, según el criterio de contenido, mediante la evaluación de juicio de seis expertos, especialistas en TIC de la PUCP. Los resultados fueron corroborados estadísticamente mediante el coeficiente $\mathrm{V}$ de Aiken, cuyo índice general fue de 0.93 , y se determinó una validez fuerte (0.91 a 1.00).
La confiabilidad del cuestionario, según el criterio de consistencia interna, se obtuvo mediante la aplicación de una prueba piloto aplicada a 66 estudiantes de la sección " $\mathrm{C}$ " de $\mathrm{III}^{\circ}, \mathrm{IV}^{\circ}$ y $\mathrm{V}^{\circ}$ de secundaria de la misma institución educativa del turno de la tarde. Posteriormente, se trabajó estadísticamente a través del coeficiente Alfa de Cronbach por cada grupo de ítems correspondiente a cada subvariable. En este 
caso, se utilizó el programa estadístico Statistical Package for the Social Sciences (SPSS), versión 21, y los resultados son los siguientes: conocimiento de las herramientas digitales (0.75), uso de las herramientas digitales (0.86), acceso y procesamiento de la información digital (0.88), ética en el uso de la información digital $(0.80)$ y comunicación en diferentes formatos digitales (0.78). Por eso, el cuestionario tiene alta confiabilidad (0.72 a 0.99$)$.

\subsection{Análisis estadístico}

La estadística utilizada en el tratamiento de la información fue descriptiva e inferencial (Vogt,
2007). Los resultados del cuestionario fueron organizados y procesados en Excel. En el caso de la estadística descriptiva, se calcularon la media y la desviación estándar. En la estadística inferencial, se aplicó la prueba Kolmogorov-Smirnov y al obtener una distribución normal de los datos, se determinó el uso de la prueba paramétrica t de Student para medias independientes y medias relacionadas para la comprobación de la hipótesis y la subhipótesis, respectivamente.

\section{Resultados}

A continuación, se presentan los resultados obtenidos en la investigación:

Tabla 3. Cuadro comparativo de las medidas de tendencia central obtenidas en el pretest y postest del grupo de control y del grupo experimental con respecto a la variable "competencia digital"

\begin{tabular}{ccccc}
\hline $\begin{array}{c}\text { Medidas de tendencia } \\
\text { central }\end{array}$ & \multicolumn{2}{c}{$\begin{array}{c}\text { Grupo de control } \\
\text { Pretest }\end{array}$} & Postest & $\begin{array}{c}\text { Competencia digital } \\
\text { Grupo experimental } \\
\text { Pretest }\end{array}$ \\
\hline Media & 1,70 & 1,79 & 1,93 & 2,09 \\
Desviación estándar & 0,51 & 0,44 & 0,34 & 0,28 \\
Total (estudiantes) & 80 & 80 & 82 & 82 \\
\hline
\end{tabular}

Fuente: elaboración propia

$\mathrm{p}<0.05$ Nivel bajo $=1$ Nivel medio $=2$ Nivel Alto $=3$

Tabla 4. Nivel de desarrollo de la competencia digital alcanzada por el grupo de control y el grupo experimental en el pretest y en el postest.

\begin{tabular}{|c|c|c|c|c|c|c|c|c|}
\hline \multirow{3}{*}{ Competencia digital } & \multicolumn{4}{|c|}{ PRETEST } & \multicolumn{4}{|c|}{ POSTEST } \\
\hline & \multicolumn{2}{|c|}{ Grupo de control } & \multicolumn{2}{|c|}{ Grupo experimental } & \multicolumn{2}{|c|}{ Grupo de control } & \multicolumn{2}{|c|}{ Grupo experimental } \\
\hline & f & $\%$ & f & $\%$ & f & $\%$ & f & $\%$ \\
\hline Nivel bajo (1) & 26 & 32,5 & 8 & 9,8 & 18 & 22,5 & 0 & 0 \\
\hline Nivel medio (2) & 52 & 65,0 & 72 & 87,8 & 61 & 76,3 & 75 & 91,5 \\
\hline Nivel bajo (3) & 2 & 2,5 & 2 & 2,4 & 1 & 1,3 & 7 & 8,5 \\
\hline Total & 80 & 100,0 & 82 & 100,0 & 80 & 100,0 & 82 & 100,0 \\
\hline
\end{tabular}

Fuente: elaboración propia

Los resultados del grupo experimental en el postest en cada subvariable son: conocimiento de las herramientas digitales (85,4\%: nivel alto), uso de las herramientas digitales (79,3\%: nivel medio), acceso y procesamiento de la información digital (79,3\%: nivel medio), ética en el uso de la información digital (58,5\%: nivel medio) y comunicación de diferentes formatos digitales (54,9\%: nivel medio). 
Tabla 5. Comparación de las medias del grupo experimental antes y después del uso de la red social educativa Edmodo

\begin{tabular}{cccccc}
\hline $\begin{array}{c}\text { Competencia digital } \\
\text { Grupo experimental }\end{array}$ & $\mathbf{N}^{\circ}$ de estudiantes & Media & $\begin{array}{c}\text { Desviación } \\
\text { estándard }\end{array}$ & t de Student & Sig. bilateral \\
\hline Post test & 82 & 106,46 & 21,485 & 4,790 & 0.000 \\
Pre test & 82 & 91,11 & 23,119 & & \\
\hline
\end{tabular}

Fuente: elaboración propia $\quad \mathrm{p}<0.05 \quad$ Nivel medio $=65-133$

Tabla 6. Comparación de las medias entre el grupo de control y grupo experimental antes del uso de la red social educativa Edmodo.

\begin{tabular}{cccccc}
\hline $\begin{array}{c}\text { Competencia digital } \\
\text { Pretest }\end{array}$ & No de estudiantes & Media & $\begin{array}{c}\text { Desviación } \\
\text { estándard }\end{array}$ & t de Student & Sig. bilateral \\
\hline Grupo experimental & 82 & 91,11 & 23,119 & & 0.002 \\
Grupo de control & 80 & 78,33 & 28,949 & 3.110 & \\
\hline
\end{tabular}

Fuente: elaboración propia $\quad \mathrm{p}<0.05 \quad$ Nivel medio $=65-133$

Tabla 7. Comparación de las medias entre el grupo de control y grupo experimental después del uso de la red social educativa Edmodo.

\begin{tabular}{cccccc}
\hline $\begin{array}{c}\text { Competencia digital } \\
\text { Postest }\end{array}$ & $\mathbf{N}^{\circ}$ de estudiantes & Media & $\begin{array}{c}\text { Desviación } \\
\text { estándard }\end{array}$ & t de Student & Sig. bilateral \\
\hline Grupo experimental & 82 & 106,46 & 21,485 & & 0.000 \\
Grupo de control & 80 & 87,75 & 26,815 & 4,908 & \\
\hline
\end{tabular}

Fuente: elaboración propia $\quad \mathrm{p}<0.05 \quad$ Nivel medio $=65-133$

\section{Discusión}

Los resultados evidencian que el nivel de desarrollo de las competencias digitales alcanzado por el grupo experimental, luego de la intervención, corresponde al nivel medio (Ver Tabla 5); es decir, el uso de las herramientas tecnológicas online y la red social educativa Edmodo han sido efectivos. Esto confirma la importancia de contextualizar las actividades en las redes sociales, aprovechando su potencial educativo para el desarrollo de competencias digitales (Vásquez, 2013). Igualmente, se corrobora la actitud positiva del docente hacia el uso de esta red social, ya que brinda espacios de trabajo colaborativo (Sáez, Lorraine y Miyata, 2013). De la misma manera, se corrobora que esta red social fomenta el trabajo colaborativo, la interactividad docente - estudiante, el intercambio de información, el refuerzo de contenidos trabajados en clase tradicional y la motivación por el trabajo en línea (Sáez, Fernández y García, 2012). Esta última investigación coincide con el presente estudio al afirmar que, a pesar de las ventajas del uso de esta red social en forma virtual, es necesario mantener el contacto presencial. Por otro lado, si bien las investigaciones anteriores se realizaron con estudiantes de secundaria, con docentes de primaria y con adultos, respectivamente, nuestro estudio amplía su uso a estudiantes de los últimos grados de secundaria. 
Analizando el promedio obtenido por el grupo de control y el grupo experimental respecto del nivel de desarrollo de las competencias digitales antes de utilizar la red social, se observó que este último alcanzó el nivel medio (Ver Tabla 6). Es decir, los estudiantes demostraron que han desarrollado, hasta cierto punto, diferentes habilidades digitales, algunas de las cuales fueron tempranamente adquiridas a partir de su interacción con la tecnología, sumado al uso de las redes sociales. Así, se confirma que este fenómeno social ha transformado las dimensiones de la comunicación y colaboración a través de internet, ya que exige utilizar recursos digitales con fines diversos a los educativos (De Haro, 2011). Asimismo, se corrobora que la ética es una dimensión del tratamiento de la información y competencia digital, en la que se desarrolla la criticidad, el uso seguro y la privacidad de los usuarios (Vivancos, 2008).

Finalmente, el promedio obtenido por el grupo experimental respecto del nivel de desarrollo de las competencias digitales luego de utilizar la red social, correspondió al nivel medio (Ver Tabla 7). Esto significa que los estudiantes manejaron las herramientas tecnológicas online y la red social. El acceso a estos recursos motivó el trabajo colaborativo de los estudiantes; así, los que tenían un nivel bajo de competencias digitales alcanzaron un nivel medio o alto de desarrollo de las mismas. Estos resultados se corroboraron con tres componentes de la competencia digital: el tecnológico, el informacional y el de ciudadanía digital (Adell, 2011). En ese sentido, el grupo experimental aprendió la utilidad de las herramientas tecnológicas de manera teórica, aunque en la práctica se evidenció cierto grado de dificultad en la elaboración de productos con estos recursos. Además, el grupo accedió con facilidad a la información digital; sin embargo, respecto al procesamiento de la misma, utilizó progresivamente las nuevas herramientas tecnológicas online. Igualmente, respecto de la ciudadanía digital, los estudiantes aprendieron a enfrentar e interactuar en entornos en los que se integraron espacios reales y virtuales. El nivel medio alcanzado por el grupo experimental reafirma que el vivir en un ambiente digital no implica ser digitalmente competentes (Li y Ranieri, 2010). En ese sentido, es necesario generalizar el desarrollo de actividades interactivas en otras áreas académicas en las que los estudiantes desarrollen la dimensión creativa, por la que se convierten en autores de contenidos (Vivancos, 2008), y en la que se convierten progresivamente de seres consumidores de información en seres productores de conocimiento, es decir, en actores sociales (Morduchowicz, 2012).

\section{CONCLUSIONES}

Al término de la aplicación del programa de intervención, los estudiantes del grupo experimental lograron un nivel de competencia digital medio, mayor que en el pretest. El mismo nivel fue alcanzado en todas las subvariables, excepto en el conocimiento de las herramientas digitales en la que llegaron al nivel alto. Así, se confirma la efectividad del uso de las herramientas tecnológicas on line y la red social educativa Edmodo en el desarrollo de las competencias digitales, lo que contribuye a formar ciudadanos que respondan a las exigencias de la sociedad de la información y la comunicación.

Por otro lado, se recomienda profundizar en el estudio de las competencias digitales para establecer una taxonomía de las mismas según el nivel educativo e integrarlas en la planificación curricular. Además, se sugiere realizar estudios cualitativos y/o mixtos en los que se investiguen las aplicaciones de otras herramientas digitales y redes sociales, así como su impacto en el trabajo colaborativo. Al llegar a este punto, es preciso reflexionar sobre cómo se relaciona el uso de las herramientas tecnológicas con los estilos de aprendizaje de los estudiantes y sobre cómo se puede evaluar el nivel de desarrollo de las competencias digitales según los estilos de aprendizaje. Por el momento, mientras encontremos respuestas a estas y otras interrogantes, se recomienda ampliar el uso de la red social educativa Edmodo a las diferentes áreas curriculares y al sexto ciclo ( $\mathrm{I}^{\circ}$ y $\mathrm{II}^{\circ}$ de secundaria), para que, de una manera gradual y contextualizada, se desarrollen las competencias digitales y se oriente el uso pedagógico de los recursos tecnológicos. Para ello, será necesario fortalecer la competencia digital docente en el uso de entornos virtuales que permitan desarrollar las competencias digitales en los estudiantes. 


\section{REFERENCIAS}

Adell, J. (2011) La competencia digital. [Audio] Tomado de: www.youtube.com/watch? $\mathrm{v}=\mathrm{tj}$ C1LOC0r1g Publicado el 13 de febrero 2011 Aguaded, J y Cabero, J. (2002) Educar en red: internet como recurso para la educación. Málaga: Aljibe

Ávalos, M. (2013). ¿Cómo integrar las TIC en la escuela del siglo XXI? De Clementina a las Tablets. Buenos Aires:Biblos.

Bisquerra, R. (1989) Métodos de Investigación Educativa. Barcelona: CEAC

Calvani, A., Fini, A., \& Ranieri, M. (2010). Digital Competence in K-12: theoretical models, assessment tools and empirical research. Anàlisi: quaderns de comunicació i cultura, (40), 157 171. Recuperado de: http://goo.gl/dsBxVd

Castañeda, L. (2010) Aprendizajes con redes sociales. Tejidos educativos para los nuevos entornos. Bogotá: MAD.

Castells, M. (2006) La era de la información: Economía, sociedad y cultura: Fin de milenio. México: Siglo XXI.

Creswell, J. (2014). Research design. Qualitative, Quantitative and Mixeed Methods Approaches. United States of America: SAGE, Publications, Inc.

De Haro, J. J. (2011). Redes sociales para la educación. Madrid: Anaya multimedia.
Ferrari, A. (2012). Digital Competence in practice: An analysis of frameworks. Joint Research Centre of the European Commission. IPTS. doi: $10.2791 / 82116$.

González, J.; Espuny, C.; de Cid, M. \& Gisbert, M. (2012). INCOTIC-ESO. Cómo autoevaluar y diagnosticar la competencia digital en la Escuela 2.0. Revista de Investigación Educativa, 30 (2), 287-302.

Hernández, R., Fernández, C. \& Baptista, M. (2010). Metodología de la investigación. México: McGraw Hill.

Jonnaert, P. (2001). Competencias y socioconstructivismo. Nuevas referencias para los programas de estudios. En Texto de apoyo a la Segunda Conferencia Anual de Inspectores de la Enseñanza Media, Bobo Dioulasso, Burkina Faso (pp. 18-22).

Li, Y. \& Ranieri, M. (2010). Are 'digital natives' really digital competent? - A study on Chinese teenagers. In British Journal of Educational Technology, 41(6), 1029-1042.

Morduchowicz, R. (2012). Los adolescentes y las redes sociales. La construcción de la identidad juvenil en Internet. Buenos Aires: Fondo de Cultura Económica. 
Sáez, J.; Fernández, M \& García, J. (2012). Descubriendo Edmodo: beneficios del microblogging en educación en adultos. Campo abierto: Revista de educación, 31(2), 53-70.

Sáez, J.; Lorraine, J. \& Miyata, Y. (2013). Uso de Edmodo en proyectos colaborativos internacionales en educación primaria. Edutec. Revista Electrónica de Tecnología Educativa, (43).

Sánchez, H. y Reyes, C. (2009). Metodología y diseños en la investigación científica. Lima: Visión Universitaria.

Siemens, G. (2004). Conectivismo: Una teoría de aprendizaje para la era digital. En R. Aparici Marino (Coord.) Conectados en el ciberespacio, Colección Grado 77 - 93

Suárez, C. (2008) Educación y virtualidad: bases para el aprendizaje cooperativo en red. Lima: Universitaria Universidad Ricardo Palma.
Vásquez, E. (2013). Microblogging con Edmodo para el desarrollo de competencias básicas del alumnado de enseñanza secundaria. Un estudio de caso. Educativo Siglo XXI, 31(1), 313-334.

Vázquez-Martínez, A. I., \& Cabero-Almenara, J. (2015). Las redes sociales aplicadas a la formación. Revista Complutense de Educación, 26, 253-272.

Vivancos, J. (2008). Tratamiento de la información y competencia digital. Madrid: Alianza Editorial, S.A.

Vogt, W. (2007). Quantitative research methods for professionals in education and other fields. Boston: Pearson Education, Inc.
Fuente de Recepción: 04 de setiembre de 2019 Fuente de Aceptación: 17 de Noviembre de 2019 